\title{
Discovery of two candidate pulsar wind nebulae in very-high-energy gamma rays
}

\author{
F. Aharonian ${ }^{1,13}$, A. G. Akhperjanian ${ }^{2}$, A. R. Bazer-Bachi ${ }^{3}$, B. Behera ${ }^{14}$, M. Beilicke $^{4}$, W. Benbow ${ }^{1}$, D. Berge ${ }^{1, \star}$, \\ K. Bernlöhr ${ }^{1,5}$, C. Boisson ${ }^{6}$, O. Bolz ${ }^{1}$, V. Borrel ${ }^{3}$, I. Braun ${ }^{1}$, E. Brion ${ }^{7}$, A. M. Brown ${ }^{8}$, R. Bühler ${ }^{1}$, I. Büsching ${ }^{9}$, \\ T. Boutelier ${ }^{17}$, S. Carrigan ${ }^{1}$, P. M. Chadwick ${ }^{8}$, L.-M. Chounet ${ }^{10}$, G. Coignet $^{11}$, R. Cornils ${ }^{4}$, L. Costamante ${ }^{1,23}$, \\ B. Degrange ${ }^{10}$, H. J. Dickinson ${ }^{8}$, A. Djannati-Atai ${ }^{12}$, W. Domainko ${ }^{1}$, L. O'C. Drury ${ }^{13}$, G. Dubus ${ }^{10}$, K. Egberts ${ }^{1}$,
} D. Emmanoulopoulos ${ }^{14}$, P. Espigat ${ }^{12}$, C. Farnier ${ }^{15}$, F. Feinstein ${ }^{15}$, A. Fiasson ${ }^{15}$, A. Förster ${ }^{1}$, G. Fontaine ${ }^{10}$, Seb. Funk ${ }^{5}$, S. Funk ${ }^{1}$, M. Füßling ${ }^{5}$, Y. A. Gallant ${ }^{15}$, B. Giebels ${ }^{10}$, J. F. Glicenstein ${ }^{7}$, B. Glück ${ }^{16}$, P. Goret ${ }^{7}$, C. Hadjichristidis ${ }^{8}$, D. Hauser ${ }^{1}$, M. Hauser ${ }^{14}$, G. Heinzelmann ${ }^{4}$, G. Henri ${ }^{17}$, G. Hermann ${ }^{1}$, J. A. Hinton ${ }^{1,14, \star \star}$, A. Hoffmann ${ }^{18}$, W. Hofmann ${ }^{1}$, M. Holleran ${ }^{9}$, S. Hoppe ${ }^{1}$, D. Horns ${ }^{18}$, A. Jacholkowska ${ }^{15}$, O. C. de Jager ${ }^{9}$, E. Kendziorra ${ }^{18}$, M. Kerschhaggl ${ }^{5}$, B. Khélifi ${ }^{10,1}$, Nu. Komin ${ }^{15}$, K. Kosack ${ }^{1}$, G. Lamanna ${ }^{11}$, I. J. Latham ${ }^{8}$, R. Le Gallou ${ }^{8}$, A. Lemière ${ }^{12}$, M. Lemoine-Goumard ${ }^{10}$, T. Lohse ${ }^{5}$, J. M. Martin ${ }^{6}$, O. Martineau-Huynh ${ }^{19}$, A. Marcowith ${ }^{3,15}$, C. Masterson ${ }^{1,23}$, G. Maurin ${ }^{12}$, T. J. L. McComb ${ }^{8}$, E. Moulin ${ }^{15,7}$, M. de Naurois ${ }^{19}$, D. Nedbal ${ }^{20}$, S. J. Nolan ${ }^{8}$, A. Noutsos ${ }^{8}$, J.-P. Olive ${ }^{3}$, K. J. Orford ${ }^{8}$, J. L. Osborne ${ }^{8}$, M. Panter ${ }^{1}$, G. Pedaletti ${ }^{14}$, G. Pelletier ${ }^{17}$, P.-O. Petrucci ${ }^{17}$, S. Pita ${ }^{12}$, G. Pühlhofer ${ }^{14}$, M. Punch $^{12}$, S. Ranchon ${ }^{11}$, B. C. Raubenheimer ${ }^{9}$, M. Raue ${ }^{4}$, S. M. Rayner ${ }^{8}$, J. Ripken ${ }^{4}$, L. Rob ${ }^{20}$, L. Rolland ${ }^{7}$, S. Rosier-Lees ${ }^{11}$, G. Rowell ${ }^{1, \star \star \star}$, J. Ruppel ${ }^{21}$, V. Sahakian ${ }^{2}$, A. Santangelo ${ }^{18}$, L. Saugé ${ }^{17}$, S. Schlenker ${ }^{5}$, R. Schlickeiser ${ }^{21}$, R. Schröder ${ }^{21}$, U. Schwanke ${ }^{5}$, S. Schwarzburg ${ }^{18}$, S. Schwemmer ${ }^{14}$, A. Shalchi ${ }^{21}$, H. Sol ${ }^{6}$, D. Spangler ${ }^{8}$, R. Steenkamp ${ }^{22}$, C. Stegmann ${ }^{16}$, G. Superina ${ }^{10}$, P. H. Tam ${ }^{14}$, J.-P. Tavernet ${ }^{19}$, R. Terrier ${ }^{12}$, M. Tluczykont ${ }^{10,23}$, $^{\text {, C. van Eldik }}{ }^{1}$, G. Vasileiadis ${ }^{15}$, C. Venter ${ }^{9}$, J. P. Vialle ${ }^{11}$, P. Vincent ${ }^{19}$, H. J. Völk ${ }^{1}$, S. J. Wagner ${ }^{14}$, and M. Ward ${ }^{8}$

(Affiliations can be found after the references)

Received 12 February 2007 / Accepted 28 April 2007

\section{ABSTRACT}

Context. We present the discovery of two very-high-energy $\gamma$-ray sources in an ongoing systematic search for emission above $100 \mathrm{GeV}$ from pulsar wind nebulae in survey data from the HESS telescope array.

Aims. Imaging Atmospheric Cherenkov Telescopes are ideal tools for searching for extended emission from pulsar wind nebulae in the very-highenergy regime. HESS, with its large field of view of $5^{\circ}$ and high sensitivity, gives new prospects for the search for these objects.

Methods. An ongoing systematic search for very-high-energy emission from energetic pulsars over the region of the Galactic plane between $-60^{\circ}<l<30^{\circ},-2^{\circ}<b<2^{\circ}$ is performed. For the resulting candidates, the standard HESS analysis was applied and a search for multiwavelength counterparts was performed.

Results. We present the discovery of two new candidate $\gamma$-ray pulsar wind nebulae, HESS J1718-385 and HESS J1809-193.

Conclusions. HESS has proven to be a suitable instrument for pulsar wind nebula searches.

Key words. gamma rays: observations - ISM: individual objects: HESS J1718 - ISM: individual objects: HESS J1809

\section{Introduction}

It has long been known that pulsars can drive powerful winds of highly relativistic particles (see e.g. Gaensler \& Slane 2006, for a review). Confinement of these winds leads to the formation of strong shocks, which may accelerate particles to $\sim \mathrm{PeV}$ energies. Evidence for high-energy electrons in pulsar wind nebulae

^ Now at CERN, Geneva, Switzerland.

$\star \star$ Now at School of Physics \& Astronomy, University of Leeds, Leeds LS2 9JT, UK.

$\star \star \star$ Now at School of Chemistry \& Physics, University of Adelaide, Adelaide 5005, Australia.

Now at DESY Zeuthen.
(PWNe) is provided by the observation of both synchrotron emission in the radio through high-energy $\gamma$-ray regimes, and by inverse Compton radiation in the high-energy and very-highenergy (VHE, $>100 \mathrm{GeV}) \gamma$-ray range. The synchrotron photon energy flux of the highly-relativistic PWN electron population is proportional to both the total number of electrons and the magnetic field energy density. The ratio of X-ray to $\gamma$-ray emission is then related to the magnetic field in the nebula. Given the general difficulty of estimating the magnetic field in such objects, it is hard to probe the spatial and energy distributions of the accelerated particles with X-ray observations alone. Measurements of high-energy $\gamma$-ray radiation resulting from inverse Compton scattering have a considerable advantage in that they provide 
Table 1. Data properties and analysis parameters for the two PWN candidates. Here, $\theta_{\mathrm{M}}$ and $\theta_{\mathrm{S}}$ are the on-source integration radii for the sky maps and the spectral analysis, respectively, $t_{\text {live }}$ is the live time, $\langle Z A\rangle$ is the mean zenith angle, $\phi$ is the mean offset of the observation position from the target position, and $E_{\text {thresh }}$ is the analysis threshold energy.

\begin{tabular}{|c|c|c|c|c|c|c|c|c|c|c|c|c|}
\hline & \multicolumn{6}{|c|}{ Sky maps } & \multicolumn{6}{|c|}{ Spectra } \\
\hline Source & $\begin{array}{l}\theta_{\mathrm{M}} \\
\left(^{\circ}\right) \\
\end{array}$ & Cuts & $\begin{array}{l}t_{\text {live }} \\
\text { (hrs) }\end{array}$ & $\begin{array}{l}\langle Z A\rangle \\
\left({ }^{\circ}\right)\end{array}$ & $\begin{array}{l}\phi \\
\left({ }^{\circ}\right)\end{array}$ & $\begin{array}{l}E_{\text {thresh }} \\
(\mathrm{GeV})\end{array}$ & & Cuts & $\begin{array}{l}t_{\text {live }} \\
(\mathrm{hrs})\end{array}$ & $\begin{array}{l}\langle Z A\rangle \\
\left(^{\circ}\right)\end{array}$ & $\begin{array}{l}\phi \\
\left({ }^{\circ}\right)\end{array}$ & $\begin{array}{l}E_{\text {thresh }} \\
(\mathrm{GeV})\end{array}$ \\
\hline HESS J1718-385 & 0.11 & hard & 82 & 33.9 & 1.6 & 450 & 0.2 & hard & 73 & 32.5 & 1.1 & 450 \\
\hline HESS J1809-193 & 0.19 & hard & 25 & 20.4 & 1.7 & 350 & 0.5 & standard & 9 & 26.5 & 1.0 & 250 \\
\hline
\end{tabular}

a direct view of the parent particle population if the target photon fields are spatially uniform and have a well-known density, e.g. where the dominant target is the cosmic microwave background radiation (CMBR) or possibly the dense interstellar radiation fields present in the inner Galaxy.

The best studied example of a PWN is the Crab nebula, which exhibits strong non-thermal emission across most of the electromagnetic spectrum from radio to $>50 \mathrm{TeV} \gamma$-rays (Hillas et al. 1998; Tanimori et al. 1998; Aharonian et al. 2004). In addition, VHE $\gamma$-rays have been detected from other young PWNe, one of them located within the supernova remnant MSH 15-52 (Aharonian et al. 2005b). More recently, VHE $\gamma$-ray emission has been detected from the Vela X PWN (Aharonian et al. 2006a). In contrast to the Crab pulsar, the Vela pulsar is an order of magnitude older ( $\sim 11 \mathrm{kyr})$ and its nebula is significantly offset from the pulsar position, both in X-rays and VHE $\gamma$-rays, possibly due to the expansion of the supernova blast wave into an inhomogeneous interstellar medium (Blondin et al. 2001). Offset nebulae in both X-rays and VHE $\gamma$-rays have also been observed in the Kookaburra Complex (Aharonian et al. 2006e) and for the PWN associated with the $\gamma$-ray source HESS J1825-137 (Aharonian et al. 2005c, 2006f). The latter source appears much brighter and more extended in VHE $\gamma$-rays than in $\mathrm{keV}$ X-rays. This suggests that searches at $\mathrm{TeV}$ energies are a powerful tool for detecting PWNe.

Motivated by these detections, an ongoing systematic search for VHE $\gamma$-ray sources associated with high spin-down energy loss rate pulsars is being performed, using data obtained with the HESS (High Energy Stereoscopic System) instrument. The HESS telescope system is currently the most sensitive instrument for $\gamma$-ray astronomy in the energy regime above $100 \mathrm{GeV}$. The instrument is an array of four $13 \mathrm{~m}$ diameter Imaging Atmospheric Cherenkov Telescopes (IACTs) located in the Khomas highland of Namibia. The system has an angular resolution better than $0.1^{\circ}$, an energy resolution of $\sim 15 \%$ and a point-source sensitivity of $<2.0 \times 10^{-13} \mathrm{~cm}^{-2} \mathrm{~s}^{-1}$ (1\% of the flux from the Crab nebula, (Aharonian et al. 2006b)) for a $5 \sigma$ detection in $25 \mathrm{~h}$ of observation. The $5^{\circ}$ field of view of the instrument and its Southern Hemisphere location make it ideal for surveying the inner Galactic plane.

The VHE $\gamma$-ray data set used in the search includes all data used in Aharonian et al. (2006c), an extension of the survey to $-60^{\circ}<l<-30^{\circ}$, dedicated observations of Galactic targets and re-observations of HESS survey sources. It spans Galactic longitudes $-60^{\circ}<l<30^{\circ}$ and Galactic latitudes $-2^{\circ}<b<2^{\circ}$, a region covered with high sensitivity in the survey. These data are being searched for VHE emission from pulsars from the Parkes Multibeam Pulsar Survey (Manchester et al. 2001). The search for a possible $\gamma$-ray excess is done in a circular region with radius $\theta=0.22^{\circ}\left(\theta=13.2^{\prime}\right)$ (as in Aharonian et al. 2006c) around each pulsar position, sufficient to encompass a large fraction of a possible PWN. The statistical significance of the resulting associations of the VHE $\gamma$-ray source with the pulsar is evaluated by repeating the procedure for randomly generated pulsar samples, modelled after the above-mentioned parent population.

In this search, it is found that pulsars with high spin-down energy loss rates are on a statistical basis accompanied by VHE emission. The VHE sources described in this paper have high statistical significances and are therefore also detected in a blind search for VHE sources (similar to that reported in Aharonian et al. 2006c).

The search for VHE $\gamma$-ray sources near the pulsars PSR J1718-3825 and PSR J1809-1917 revealed two new VHE $\gamma$-ray sources, HESS J1718-385 and HESS J1809-193, respectively. This paper deals with the results from the HESS data analysis of these two new sources, and with their possible associations with the pulsars and other objects seen in radio and $\mathrm{X}$-ray wavelengths. In the data set used for this search, other new VHE sources have been detected that are not associated with pulsars, and will be reported elsewhere.

\section{HESS observations and analysis}

The data on the first source, HESS J1718-385, are composed primarily from dedicated observations of the supernova remnant RX J1713.7-3946 (G347.3-0.5), which is located 1.6 $6^{\circ}$ southwest of HESS J1718-385 (Koyama et al. 1997; Muraishi et al. 2000; Aharonian et al. 2006d). The first HESS observations of the region around the second source, HESS J1809-193, were taken as part of the systematic survey of the inner Galaxy from May to June 2004 (Aharonian et al. 2005a, 2006c). As there was a marginally significant VHE $\gamma$-ray signal ( $2 \sigma$ post-trials $)$, reobservations of HESS J1809-193 were taken in 2004 and 2005. After passing the HESS standard data quality criteria (Aharonian et al. 2006b) based on hardware and weather conditions, the data set for HESS J1718-385 has a total live time of $\sim 82 \mathrm{~h}$ and for HESS J1809-193 a live time of $\sim 25 \mathrm{~h}$ (see Table 1 for observation properties and analysis parameters for both sources). Both data sets come from observations whose centre positions are less than $3^{\circ}$ offset from the respective best-fit source position. Since most of the observations were not specifically targeted at these sources, offsets from the pointing positions are larger than for dedicated observations (which are typically offset by $\leq 1.0^{\circ}$ ).

The standard HESS analysis scheme (Aharonian et al. 2006b) was applied to the data, including optical efficiency corrections. The off-axis sensitivity of the system derived from Monte Carlo simulations and observational data has been confirmed by observations of the Crab nebula. Cuts on the scaled width and length of images (optimised on $\gamma$-ray simulations and off-source data) were used to suppress the hadronic background.

To produce sky maps, hard cuts (Aharonian et al. 2006b) were applied, which include a rather tight cut on the shower image brightness of 200 photo-electrons and are suitable for extended, hard-spectrum sources such as PWN. The background at each test position in the sky was derived from a ring with a mean radius of 1 degree surrounding this position and a width scaled 

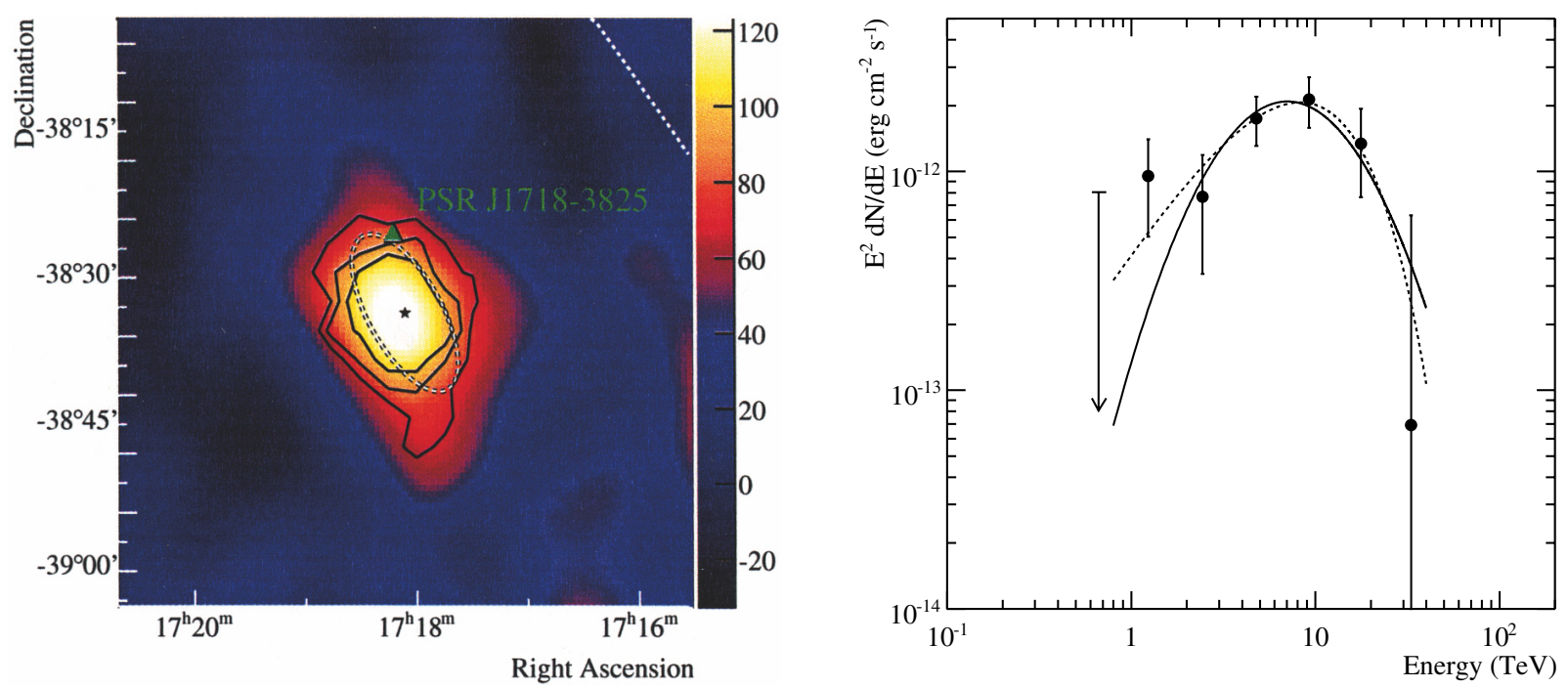

Fig. 1. Left: an image of the VHE $\gamma$-ray excess counts of HESS J1718-385, smoothed with a Gaussian of width $3.8^{\prime}$. The colour scale is set such that the blue/red transition occurs at approximately the $3 \sigma$ significance level. The black contours are the 4,5 and $6 \sigma$ significance contours. For this significance calculation, events were correlated with a top hat function with an rms chosen to match that of the Gaussian smoothing (and thus an integration radius $\theta_{\mathrm{M}}=6.6^{\prime}$ ). The position of the pulsar PSR J1718-3825 is marked with a green triangle and the Galactic plane is shown as a white dotted line. The best fit position for the $\gamma$-ray source is marked with a black star and the fit ellipse with a dashed line. Right: the energy spectrum of HESS J1718-385, which is fit by a curved profile (solid line). Alternatively, the fit of an exponentially cut-off power law is shown (dashed line, refer to the text for details on both fits). The first point in the spectrum lacks statistics due to lower exposure at small zenith angles and is plotted as a $2 \sigma$ upper limit.

to provide a background area $\sim 7$ times larger than the area of the on-source region, which is defined as a circle of radius $\theta_{\mathrm{M}}$.

For spectral studies, only observations in which the camera centre is offset by less than $2^{\circ}$ from the respective best-fit source positions were used to reduce systematic effects due to reconstructed $\gamma$-ray positions falling close to edge of the field of view. The spectral significances are calculated by counting events within a circle of radius $\theta_{\mathrm{S}}$ from the best fit position. The integration radius was chosen to enclose the whole emission region for each source while reducing systematic effects arising from morphology assumptions. In the case of HESS J1809-193, standard cuts (Aharonian et al. 2006b) on the image parameters were applied, loosening the minimum image brightness to 80 photo-electrons to extend the energy spectrum down to $\sim 250 \mathrm{GeV}$ (at the expense of a reduced signal-to-noise ratio), and the background was estimated from regions with equal offset from the centre of the field of view to minimise spectral systematic uncertainties. For HESS J1718-385, hard cuts were retained for deriving the energy spectrum as these cuts also improve the angular resolution and therefore suppress contamination from the nearby supernova remnant RX J1713.7-3946. The proximity of this strong source made it necessary to choose the background data from off-source observations (matched to the zenith angle and offset distribution of the on-source data) instead of from areas in the same field of view. For a more detailed description of methods for background estimation, see Berge et al. (2007). The remaining live times of the data samples were $\sim 9 \mathrm{~h}$ in case of HESS J1809-193 and $\sim 73 \mathrm{~h}$ in case of HESS J1718-385.

\section{Results}

\subsection{HESS J1718-385}

The detection significance from the search for VHE $\gamma$-ray emission within $13.2^{\prime}$ of the location of PSR J1718-3825 is $7.9 \sigma$. A very conservative estimate of the number of trials involved (similar to Aharonian et al. 2006c) leads to a corrected significance of $6.2 \sigma$.

The left panel of Fig. 1 shows the excess count map of the $60^{\prime} \times 60^{\prime}$ region around HESS J1718-385, smoothed with a Gaussian of width $3.8^{\prime}$ chosen to reduce statistical fluctuations while retaining source features. A two-dimensional Gaussian brightness profile, folded with the HESS point-spread function, is fit to the distribution before smoothing. Its parameters are the width in two dimensions and the position angle, defined counterclockwise from North. The intrinsic widths (with the effect of the point-spread function removed) for the fit are $9^{\prime} \pm 2^{\prime}$ and $4^{\prime} \pm 1^{\prime}$ and the position angle is $\sim 33^{\circ}$. The best fit position for the centre of the excess is RA $=17^{\mathrm{h}} 18^{\mathrm{m}} 7^{\mathrm{s}} \pm 5^{\mathrm{s}}$, Dec $=-38^{\circ} 33^{\prime} \pm 2^{\prime}$ (epoch J2000). HESS has a systematic pointing error of $\sim 20^{\prime \prime}$.

For the spectral analysis, a statistical significance of $6.8 \sigma$ (with 343 excess counts) was derived. Figure 1 (right) shows the measured spectral energy distribution for HESS J1718-385 (in $E^{2} \mathrm{~d} N / \mathrm{d} E$ representation). The fit of a pure power law $(\mathrm{d} N / \mathrm{d} E=$ $\left.N_{0} E^{-\Gamma}\right)$ to the spectrum gives a $\chi^{2} /$ d.o.f. $=11.1 / 4$ and a spectral index of $\Gamma=2.1 \pm 0.1_{\text {stat }} \pm 0.2_{\text {sys }}$. line):

The spectrum is fit by a curved profile (shown as the solid

$$
\frac{\mathrm{d} N}{\mathrm{~d} E}=N_{0}\left(\frac{E_{\text {peak }}}{1 \mathrm{TeV}}\right)^{-2}\left(\frac{E}{E_{\text {peak }}}\right)^{\beta \cdot \ln \left(E / E_{\text {peak }}\right)-2.0} .
$$

The peak energy $E_{\text {peak }}$ is $\left(7 \pm 1_{\text {stat }} \pm 1_{\text {sys }}\right) \mathrm{TeV}$, the differential flux normalisation $N_{0}=\left(1.3 \pm 0.3_{\text {stat }} \pm 0.5_{\text {sys }}\right) \times$ $10^{-12} \mathrm{TeV}^{-1} \mathrm{~cm}^{-2} \mathrm{~s}^{-1}$ and $\beta=-0.7 \pm 0.3_{\text {stat }} \pm 0.4_{\text {sys. }}$. This fit has a $\chi^{2} /$ d.o.f. $=3.2 / 3$. The integral flux between $1-10 \mathrm{TeV}$ is about $2 \%$ of the flux of the Crab nebula in the same energy range (Aharonian et al. 2006b). This spectral fit was used to derive the energy flux used later in Table 2 .

Alternatively, fitting the spectrum by an exponentially cut-off power law $\left(\mathrm{d} N / \mathrm{d} E=N_{0} E^{-\Gamma} \mathrm{e}^{-E / E_{\text {cut }}}\right)$ gives $N_{0}=$ $\left(3.0 \pm 1.9_{\text {stat }} \pm 0.9_{\text {sys }}\right) \times 10^{-13} \mathrm{TeV}^{-1} \mathrm{~cm}^{-2} \mathrm{~s}^{-1}$, photon index 
Table 2. HESS J1718-385 and HESS J1809-193 as pulsar wind nebulae: summary of the properties of the possibly associated pulsars, PSR J1718-3825 and PSR J1809-1917, respectively, taken from Manchester et al. (2005). The spin-down age, defined as $P / 2 \dot{P}$ (where $P$ is the period of the pulsar), provides an age estimate for a pulsar if the birth period of the pulsar was short in comparison to the current period and assuming a braking index $n=3$ (Kramer et al. 2003). The pulsar distances result from Taylor \& Cordes (1993); a more recent estimate for the distances of the pulsars from Cordes \& Lazio (2002) (given in parentheses) does not significantly change the estimates for size, offset and efficiency. The size refers to the larger intrinsic width from the respective fits (see Sect. 3.1), and the offset refers to the distance of the pulsar from the best fit position of the VHE emission region in each case. The efficiency, $\epsilon_{\gamma}$, is defined as the ratio of the $\gamma$-ray luminosity of the VHE source to the spin-down power of the pulsar.

\begin{tabular}{lllllllll}
\hline \hline Pulsar & $\begin{array}{l}\text { Spin-down } \\
\text { luminosity } \\
\left(\mathrm{erg} \mathrm{s}^{-1}\right)\end{array}$ & $\begin{array}{l}\text { Spin-down } \\
\text { age } \\
(\mathrm{kyr})\end{array}$ & $\begin{array}{l}\text { Distance } \\
(\mathrm{kpc})\end{array}$ & Source & $\begin{array}{l}1-10 \mathrm{TeV} \\
\text { Flux } \\
\left(\mathrm{erg} \mathrm{cm}^{-2} \mathrm{~s}^{-1}\right)\end{array}$ & $\begin{array}{l}\text { Size } \\
(\mathrm{pc})\end{array}$ & $\begin{array}{l}\text { Offset } \\
(\mathrm{pc})\end{array}$ & $\begin{array}{l}\epsilon_{\gamma} \\
(1-10 \mathrm{TeV})\end{array}$ \\
\hline $\mathrm{JSR}$ & $1.3 \times 10^{36}$ & 90 & $4.2(3.6)$ & $\mathrm{J} 1718-385$ & $2.9 \times 10^{-12}$ & 11 & 10 & $0.5 \%$ \\
$\mathrm{~J} 1809-1917$ & $1.8 \times 10^{36}$ & 51 & $3.7(3.5)$ & $\mathrm{J} 1809-193$ & $1.3 \times 10^{-11}$ & 35 & 13 & $1.2 \%$ \\
\hline
\end{tabular}
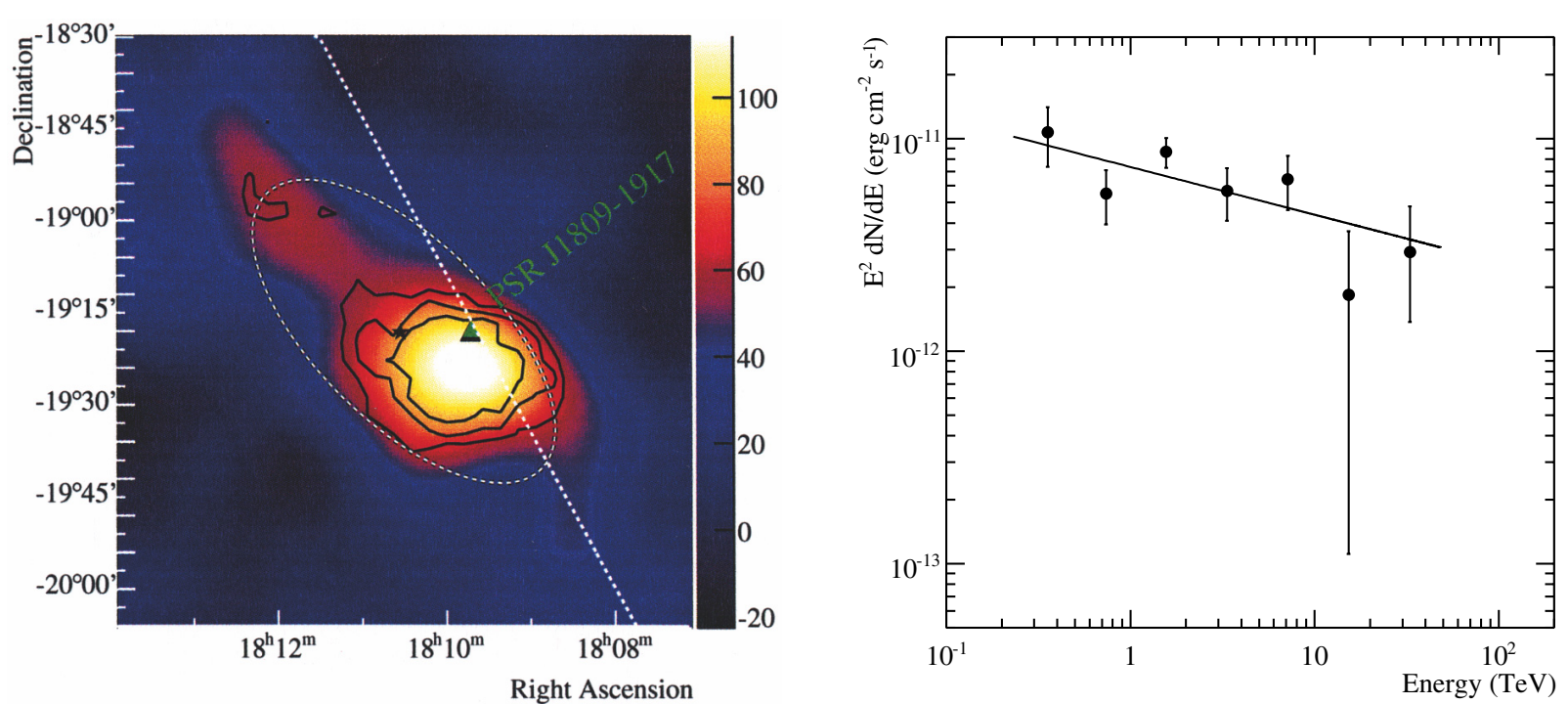

Fig. 2. Left: an image of the VHE $\gamma$-ray excess counts of HESS J1809-193, smoothed with a Gaussian of width 6.6' ${ }^{\prime}$ The colour scale is set such that the blue/red transition occurs at approximately the $3 \sigma$ significance level. The black contours are the 4,5 and $6 \sigma$ significance contours. For this significance calculation, events were correlated with a top hat function with an rms chosen to match that of the Gaussian smoothing (and thus an integration radius $\theta_{\mathrm{M}}=11.4^{\prime}$ ). The position of the pulsar PSR J1809-1917 is marked with a green triangle, while the Galactic plane is shown as a white dotted line. The best fit position for the $\gamma$-ray source is marked with a black star and the fit ellipse with a dashed line. Right: the energy spectrum of HESS J1809-193, which is fit by a power law with slope $\Gamma=2.2 \pm 0.1_{\text {stat }} \pm 0.2_{\text {sys }}$.

$\Gamma=0.7 \pm 0.6_{\text {stat }} \pm 0.2_{\text {sys }}$ and a cut-off in the spectrum at an energy of $E_{\text {cut }}=\left(6 \pm 3_{\text {stat }} \pm 1_{\text {sys }}\right) \mathrm{TeV}$. This fit, which is shown as a dashed line in the right panel of Fig. 1, has a $\chi^{2} /$ d.o.f. $=1.6 / 3$. The integral flux between $1-10 \mathrm{TeV}$ is about $2 \%$ of the flux of the Crab nebula in the same energy range (Aharonian et al. 2006b).

Both the curved and exponentially cut-off power-law profiles fit the data well; the former has the advantage of showing explicitely the peak energy of the spectrum, which has to date only been resolved in one other VHE source, Vela X (Aharonian et al. 2006a).

\subsection{HESS J1809-193}

The detection significance from the search for VHE $\gamma$-ray emission within $13.2^{\prime}$ of the location of PSR J1809-1917 is $6.8 \sigma$. A very conservative estimate of the number of trials involved (as in Aharonian et al. 2006c) leads to a corrected significance of $4.7 \sigma$. In Fig. 2, the left panel displays the uncorrelated excess count map of the $90^{\prime} \times 90^{\prime}$ region around HESS J1809-193, smoothed with a Gaussian of width $6.6^{\prime}$, again chosen to reduce statistical fluctuations while retaining source features.
Again assuming a two-dimensional Gaussian brightness profile folded with the HESS point-spread function for this clearly extended source, the intrinsic widths of the fit ellipse are $32^{\prime} \pm$ $4^{\prime}$ and $15^{\prime} \pm 2^{\prime}$, and the position angle is $\sim 50^{\circ}$. The best fit position for the centre of the excess is RA $=18^{\mathrm{h}} 10^{\mathrm{m}} 31^{\mathrm{s}} \pm 12^{\mathrm{s}}$, Dec $=$ $-19^{\circ} 18^{\prime} \pm 2^{\prime}$ (epoch J2000).

For the spectral analysis, a statistical significance of $7.6 \sigma$ (with 875 excess counts) was derived. The right panel of Fig. 2 shows the measured spectral energy distribution for HESS $\mathrm{J} 1809-193$ (in $E^{2} \mathrm{~d} N / \mathrm{d} E$ representation). The spectrum is well fit by a power law $\left(\mathrm{d} N / \mathrm{d} E=N_{0} E^{-\Gamma}\right)$ with photon index $\Gamma=2.2 \pm 0.1_{\text {stat }} \pm 0.2_{\text {sys }}$ and differential flux normalisation at $1 \mathrm{TeV} N_{0}=\left(4.6 \pm 0.6_{\text {stat }} \pm 1.4_{\text {sys }}\right) \times 10^{-12} \mathrm{TeV}^{-1} \mathrm{~cm}^{-2} \mathrm{~s}^{-1}$ and gives a $\chi^{2} /$ d.o.f. $=6.9 / 5$. Its integral flux between $1-10 \mathrm{TeV}$ is about $14 \%$ of the flux of the Crab nebula in the same energy range.

\section{Possible associations}

In an ongoing search for possible associations of energetic pulsars with VHE emission, measured with HESS in the Galactic plane, it is found that both new sources presented in this paper are each plausibly associated with such a pulsar. 

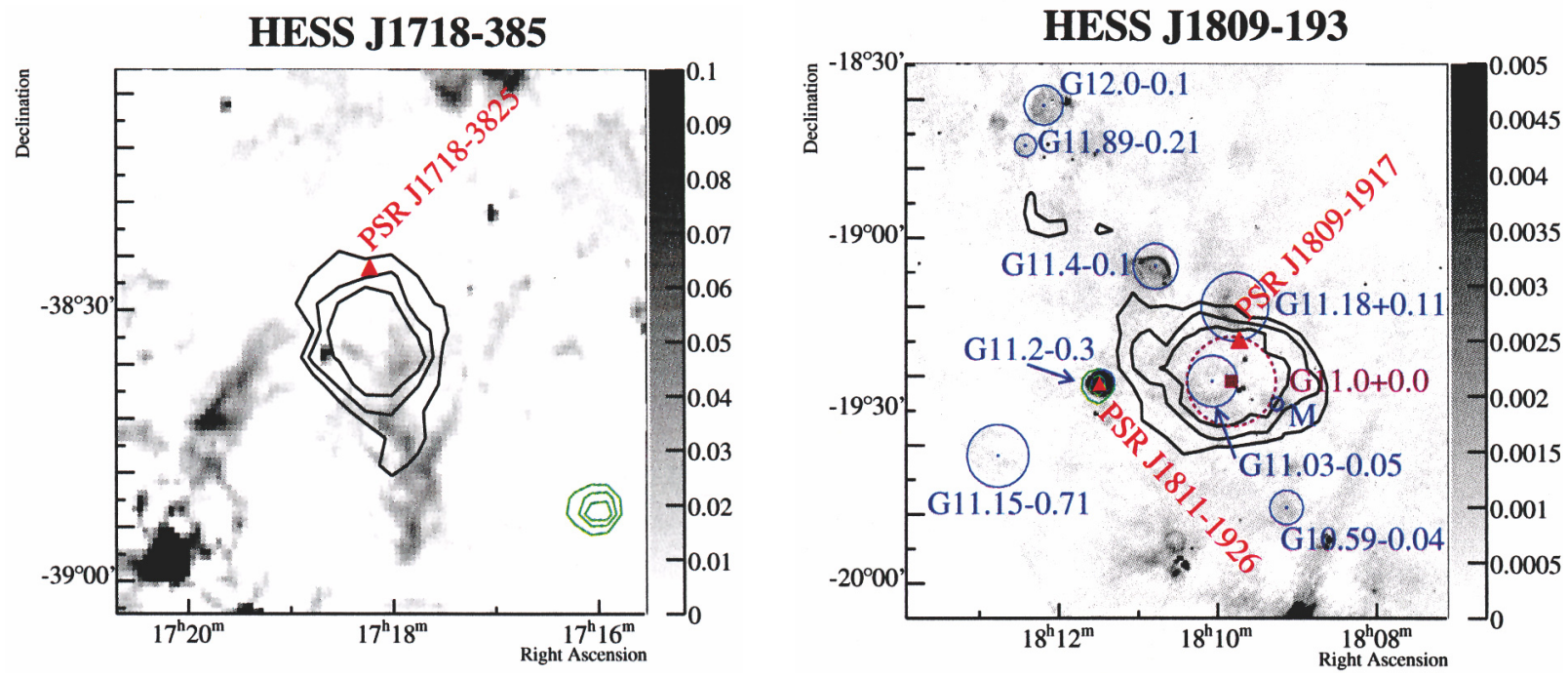

Fig. 3. Radio images from the Molonglo Galactic Plane Survey at $843 \mathrm{MHz}$ (Green et al. 1999) in the case of HESS J1718-385 in the left panel, and from the MAGPIS survey at $1.4 \mathrm{GHz}$ (Helfand et al. 2006) in the case of HESS J1809-193 in the right panel (both in Jy/beam). The HESS significance contours are overlaid in black and pulsar positions are marked with red triangles. Adaptively smoothed ROSAT hard-band $\mathrm{X}$-ray contours are shown in green (Voges et al. 2001). Blue circles in the image of the HESS J1809-193 region indicate catalogued positions and sizes of nearby supernova remnants (Green 2004) and supernova remnant candidates (Brogan et al. 2006). The position of the ASCA source G11.0+0.0 is marked with a magenta square and its size is indicated with a dashed magenta circle (Bamba et al. 2003). The most prominent source in the ROSAT data is the supernova remnant G11.2-0.3, which surrounds the X-ray emitting pulsar PSR J1811-1925 (Kaspi et al. 2001). The MAGPIS supernova remnant candidate $10.8750+0.0875$ is labeled $M$ (Helfand et al. 2006).

The properties of both pulsars, which were discovered in the Parkes Multibeam Pulsar Survey (Manchester et al. 2001; Morris et al. 2002) are summarised in Table 2. In this section, these probable associations are investigated by evaluating broad-band information around the objects.

Both PSR J1718-3825 and PSR J1809-1917 appear to be Vela-like pulsars, as they are of comparable age and show similar spin periods, $75 \mathrm{~ms}$ and $83 \mathrm{~ms}$ for PSR J1718-3825 and PSR J1809-1917, respectively. Compared with the general sample used in the systematic search, the associated VHE emission seems to fit into the emerging picture of $\gamma$-ray PWNe, as implied by sources like Vela X, HESS J1825-137 and the two PWN candidates in the Kookaburra complex. The offset of the VHE emission from the pulsar position is not atypical compared with other probable PWNe associations. Such offset PWNe can be explained by either the proper motion of the pulsar, or by interaction with non-uniform regions in the ISM (Blondin et al. 2001).

The $\gamma$-ray source HESS J1718-385 is located $\sim 0.14^{\circ}$ south of the pulsar PSR J1718-3825 (shown as a green triangle in Fig. 1 and as a red triangle in the left panel of Fig. 3). With a distance of $\sim 4 \mathrm{kpc}$ and a spin-down flux $\dot{E} / d^{2}$ on the order of $10^{35} \mathrm{erg} \mathrm{kpc}^{-2} \mathrm{~s}^{-1}$, the pulsar is energetic enough to power HESS J1718-385, with an implied efficiency of conversion into $1-10 \mathrm{TeV} \gamma$-rays of $\epsilon_{\gamma}=0.5 \%$.

As can be seen in the left panel of Fig. 3, no obvious X-ray counterpart is visible for HESS J1718-385. There is diffuse extended radio emission, which is partially coincident with the VHE emission, however, this emission seems to be correlated with thermal dust emission visible in the IRAS Sky Survey Atlas (Neugebauer et al. 1984), suggesting that the radio emission is thermal and is thus not likely associated with a possible PWN. The brightest part of this diffuse feature is catalogued as PMN J1717-3846 (Wright et al. 1994). From the point of view of positional coincidence, energetics, and lack of other counterparts, the association of HESS J1718-385 with PSR J1718-3825 seems plausible. To confirm this, additional evidence from spectral and morphological studies in VHE $\gamma$-rays and from data at other wavelengths is needed.

PSR J1718-3825 has so far no associated PWN detected in radio to X-ray wavelengths. Since the VHE emission is assumed to come from inverse Compton scattering of high-energy electrons off the CMBR, the VHE spectrum predicts the electron spectrum and one would expect to see X-ray synchrotron emission from this object. Under the assumption of a magnetic field in the range of a few $\mu \mathrm{G}$, the expected peak synchrotron flux is $\sim 10^{-12}(B / 5 \mu \mathrm{G})^{2} \mathrm{erg} \mathrm{cm}^{-2} \mathrm{~s}^{-1}$. It should be possible for instruments such as XMM-Newton to reveal an X-ray counterpart. However, with the cut-off seen at $\sim 10 \mathrm{TeV}$ in VHE $\gamma$-rays, the $\mathrm{X}$-ray flux may peak below the XMM-Newton range.

The best fit position of the second new VHE $\gamma$-ray source, HESS J1809-193, is located at a distance of $\sim 0.2^{\circ}$ east of the pulsar PSR J1809-1917, while the peak of the VHE emission is at $\sim 0.2^{\circ}$ to the south of the pulsar. Its spin-down flux $\dot{E} / d^{2}$, on the order of $10^{35} \mathrm{erg} \mathrm{kpc}^{-2} \mathrm{~s}^{-1}$, implies an efficiency of $\epsilon_{\gamma}=1.2 \%$, assuming it powers the whole emission from HESS J1809-193 (see Table 2).

In the case of HESS J1809-193, the multi-wavelength picture is much more complicated than for HESS J1718-385. The right panel of Fig. 3 shows the diffuse ASCA source G11.0+0.0 (Bamba et al. 2003; Brogan et al. 2004, 2006) coinciding with the peak of the VHE emission, which makes it a possible Xray counterpart to HESS J1809-193. The flux of this X-ray source is $\sim 3.8 \times 10^{-12} \mathrm{erg} \mathrm{cm}^{-2} \mathrm{~s}^{-1}$ and its photon index $\sim 1.6$ in the energy range $0.7-10 \mathrm{keV}$. Based on its X-ray spectrum, it was suggested that this source could be a plerionic supernova remnant. The source distance is estimated to be $2.6 \mathrm{kpc}$, while PSR J1809-1917 is estimated to be $3.5 \mathrm{kpc}$ away, however, this does not rule out the association between the ASCA source and PSR J1809-1917 as both distance estimates suffer from large 
systematic uncertainties. A further hint for the VHE emission coming from a PWN is found in public Chandra data. There appears to be a PWN visible coincident with PSR J1809-1917, that apparently shows a cometary "tail" structure near the position of the pulsar, on a much smaller scale than the $\gamma$-ray emission (Sanwal et al. 2005). Future X-ray observations should give more insight into its morphology.

The most prominent source in the ROSAT data is the supernova remnant G11.2-0.3, which lies just outside the significant VHE emission region and surrounds the X-ray emitting pulsar PSR J1811-1925 (marked with a red triangle). As the pulsar is associated with G11.2-0.3, which is estimated to be at a distance of $\sim 4.4 \mathrm{kpc}$, it is powerful enough to emit VHE $\gamma$-rays, as its spin-down flux is then $3.3 \times 10^{35} \mathrm{erg} \mathrm{kpc}^{-2} \mathrm{~s}^{-1}$. However, in this case, an association with the HESS source is highly unlikely. As Chandra has revealed, the pulsar is very close $\left(\leq 8^{\prime \prime}\right)$ to the geometric centre of the shell and its PWN is shown to lie within the supernova remnant (Kaspi et al. 2001). Thus it seems improbable that PSR J1811-1925 could have produced an extremely offset VHE PWN due to its motion, as it seems to not yet have left its supernova remnant shell.

Finally, PSR J1809-1917 might also be associated with the supernova remnant candidates G11.03-0.05, G11.18+0.11 (Brogan et al. 2004, 2006), or with the MAGPIS supernova remnant candidate $10.8750+0.0875$ (Helfand et al. 2006), which are all located within the VHE emission region. Given the small angular sizes of these supernova remnants, none of them is likely to be responsible for the bulk of the VHE emission.

\section{Summary}

HESS J1718-385 might represent the first VHE $\gamma$-ray PWN found in a systematic search for pulsar associations, despite the present lack of a PWN detection in other wavebands. The remarkable similarity between HESS J1718-385 and other known VHE PWNe, together with the lack of other probable counterparts, gives additional confidence. The detection of an X-ray PWN would provide confirmation.

In the case of HESS J1809-193 the multi-wavelength picture is much more complicated. Though the bulk of the emission can be explained by a PWN powered by PSR J1809-1917, several supernova remnants as well as another PWN may contribute to the observed VHE emission. Deeper observations in both $\gamma$-ray and X-ray wavebands are needed to conclusively distinguish the origin of the signal.

With the increasing number of detections of PWNe and PWN candidates powered by high spin-down flux pulsars, VHE $\gamma$-rays are likely to be a useful tool for discovering more of these types of objects.

Acknowledgements. The support of the Namibian authorities and of the University of Namibia in facilitating the construction and operation of HESS is gratefully acknowledged, as is the support by the German Ministry for Education and Research (BMBF), the Max Planck Society, the French Ministry for Research, the CNRS-IN2P3 and the Astroparticle Interdisciplinary Programme of the CNRS, the UK Particle Physics and Astronomy Research Council. (PPARC), the IPNP of the Charles University, the South African Department of Science and Technology and National Research Foundation, and by the University of Namibia. We appreciate the excellent work of the technical support staff in Berlin, Durham, Hamburg, Heidelberg, Palaiseau, Paris, Saclay, and in Namibia in the construction and operation of the equipment.

We thank M. Ostrowski for useful comments.

We have made use of the ROSAT Data Archive of the Max-Planck-Institut fuer extraterrestrische Physik (MPE) at Garching, Germany.

\section{References}

Aharonian, F., Akhperjanian, A., Beilicke, M., et al. 2004, ApJ, 614, 897 Aharonian, F., Akhperjanian, A. G., Aye, K.-M., et al. 2005a, Science, 307, 1938 Aharonian, F., Akhperjanian, A. G., Aye, K.-M., et al. 2005b, A\&A, 435, L17 Aharonian, F. A., Akhperjanian, A. G., Bazer-Bachi, A. R., et al. 2005c, A\&A, 442, L25

Aharonian, F., Akhperjanian, A. G., Bazer-Bachi, A. R., et al. 2006a, A\&A, 448, L43

Aharonian, F., Akhperjanian, A. G., Bazer-Bachi, A. R., et al. 2006b, A\&A, 457, 899

Aharonian, F., Akhperjanian, A. G., Bazer-Bachi, A. R., et al. 2006c, ApJ, 636, 777

Aharonian, F., Akhperjanian, A. G., Bazer-Bachi, A. R., et al. 2006d, A\&A, 449, 223

Aharonian, F., Akhperjanian, A. G., Bazer-Bachi, A. R., et al. 2006e, A\&A, 456, 245

Aharonian, F., Akhperjanian, A. G., Bazer-Bachi, A. R., et al. 2006f, A\&A, 460, 365

Bamba, A., Ueno, M., Koyama, K., \& Yamauchi, S. 2003, ApJ, 589, 253

Berge, D., Funk, S., \& Hinton, J. 2007, A\&A, 466, 1219

Blondin, J. M., Chevalier, R. A., \& Frierson, D. M. 2001, ApJ, 563, 806

Brogan, C. L., Devine, K. E., Lazio, T. J., et al. 2004, AJ, 127, 355

Brogan, C. L., Gelfand, J. D., Gaensler, B. M., Kassim, N. E., \& Lazio, T. J. W. 2006, ApJ, 639, L25

Cordes, J. M., \& Lazio, T. J. W. 2002, [arXiv: astro-ph/0207156]

Gaensler, B. M., \& Slane, P. O. 2006, ARA\&A, 44, 17

Green, A. J., Cram, L. E., Large, M. I., \& Ye, T. 1999, ApJS, 122, 207

Green, D. A. 2004, Bull. Astron. Soc. Ind., 32, 335

Helfand, D. J., Becker, R. H., White, R. L., Fallon, A., \& Tuttle, S. 2006, AJ, 131,2525

Hillas, A. M., Akerlof, C. W., Biller, S. D., et al. 1998, ApJ, 503, 744

Kaspi, V. M., Roberts, M. E., Vasisht, G., et al. 2001, ApJ, 560, 371

Koyama, K., Kinugasa, K., Matsuzaki, K., et al. 1997, PASJ, 49, L7

Kramer, M., Lyne, A. G., Hobbs, G., et al. 2003, ApJ, 593, L31

Manchester, R. N., Lyne, A. G., Camilo, F., et al. 2001, MNRAS, 328, 17

Manchester, R. N., Hobbs, G. B., Teoh, A., \& Hobbs, M. 2005, AJ, 129, 1993

Morris, D. J., Hobbs, G., Lyne, A. G., et al. 2002, MNRAS, 335, 275

Muraishi, H., Tanimori, T., Yanagita, S., et al. 2000, A\&A, 354, L57

Neugebauer, G., Habing, H. J., van Duinen, R., et al. 1984, ApJ, 278, L1

Sanwal, D., Pavlov, G. G., \& Garmire, G. P. 2005, BAAS, 37, 497

Tanimori, T., Sakurazawa, K., Dazeley, S. A., et al. 1998, ApJ, 492, L33

Taylor, J. H., \& Cordes, J. M. 1993, ApJ, 411, 674

Voges, W., Boller, T., Englhauser, J., Freyberg, M., \& Supper, R. 2001, in Virtual Observatories of the Future, ed. R. J. Brunner, S. G. Djorgovski, \& A. S. Szalay, ASP Conf. Ser., 225, 234

Wright, A. E., Griffith, M. R., Burke, B. F., \& Ekers, R. D. 1994, ApJS, 91, 111

1 Max-Planck-Institut für Kernphysik, PO Box 103980, 69029 Heidelberg, Germany

e-mail: [svenja.carrigan; karl . kosack]@mpi-hd.mpg.de

2 Yerevan Physics Institute, 2 Alikhanian Brothers St., 375036 Yerevan, Armenia

3 Centre d'Étude Spatiale des Rayonnements, CNRS/UPS, 9 Av. du Colonel Roche, BP 4346, 31029 Toulouse Cedex 4, France

4 Universität Hamburg, Institut für Experimentalphysik, Luruper Chaussee 149, 22761 Hamburg, Germany

5 Institut für Physik, Humboldt-Universität zu Berlin, Newtonstr. 15, 12489 Berlin, Germany

6 LUTH, UMR 8102 du CNRS, Observatoire de Paris, Section de Meudon, 92195 Meudon Cedex, France

7 DAPNIA/DSM/CEA, CE Saclay, 91191 Gif-sur-Yvette, Cedex, France

8 University of Durham, Department of Physics, South Road, Durham DH1 3LE, UK

9 Unit for Space Physics, North-West University, Potchefstroom 2520, South Africa

${ }^{10}$ Laboratoire Leprince-Ringuet, IN2P3/CNRS, École Polytechnique, 91128 Palaiseau, France

11 Laboratoire d'Annecy-le-Vieux de Physique des Particules, IN2P3/CNRS, 9 chemin de Bellevue, BP 110, 74941 Annecy-leVieux Cedex, France

12 APC, UMR 7164 (CNRS, Université Paris VII, CEA, Observatoire de Paris), 11 place Marcelin Berthelot, 75231 Paris Cedex 05, France 
13 Dublin Institute for Advanced Studies, 5 Merrion Square, Dublin 2, Ireland

${ }^{14}$ Landessternwarte, Universität Heidelberg, Königstuhl, 69117 Heidelberg, Germany

15 Laboratoire de Physique Théorique et Astroparticules, IN2P3/CNRS, Université Montpellier II, CC 70, Place Eugène Bataillon, 34095 Montpellier Cedex 5, France

16 Universität Erlangen-Nürnberg, Physikalisches Institut, ErwinRommel-Str. 1, 91058 Erlangen, Germany

17 Laboratoire d'Astrophysique de Grenoble, INSU/CNRS, Université Joseph Fourier, BP 53, 38041 Grenoble Cedex 9, France
18 Institut für Astronomie und Astrophysik, Universität Tübingen, Sand 1, 72076 Tübingen, Germany

19 Laboratoire de Physique Nucléaire et de Hautes Énergies, IN2P3/CNRS, Universités Paris VI \& VII, 4 place Jussieu, 75252 Paris Cedex 5, France

20 Institute of Particle and Nuclear Physics, Charles University, V Holesovickach 2, 18000 Prague 8, Czech Republic

${ }^{21}$ Institut für Theoretische Physik, Lehrstuhl IV: Weltraum und Astrophysik, Ruhr-Universität Bochum, 44780 Bochum, Germany 22 University of Namibia, Private Bag 13301, Windhoek, Namibia ${ }^{23}$ European Associated Laboratory for Gamma-Ray Astronomy, jointly supported by CNRS and MPG 